Colorectal cancer is one of the most often diagnosed malignant tumors. In Kazakhstan, high incidence of CC is registered along with other oncology diseases. Despite a significant progress in the disease treatment achieved lately, CC is still one of the major reasons of mortality due to oncologic pathologies.

To study the samples MilliplexMap HumanCirculationBiomarker panel in blood serum was used. XMap-based Fluorescence immunoassay was implemented, which comprised magnetic-bead-based simultaneous fluorescence detection of IL-6, IL-8, MIF, FGF-2, SCF, TGF, TNF, TRAIL analytes. Proinflammatory biomarker concentration detection at different CC stages allows to reveal the dynamics of inflammatory response of the organism to tumor and to use them (biomarkers) in further diagnostic and forecast in particular in CC. As a result of our study, it was found that IL-6, which showed the brightest reaction, due to its range of change and considerable shift already in the I stage can be recommended as a component of a complex diagnostic panel. Such markers as FGF2 and MIF also have a role in CC early stage detection.

Key words: cancer biomarkers, cytokine, colorectal cancer, inflammation, interleukin.

Contemp Oncol (Pozn) 2020; 24 (4): 207-210 DOI: https://doi.org/10.5114/wo.2020.102551

\section{Proinflammatory cytokines and colorectal cancer - the impact of the stage}

\author{
Lyudmila Akhmaltdinova, Valentina Sirota, Dmitriy Babenko, \\ Vera Zhumaliyeva, Irina Kadyrova, Mereke Maratkyzy, Aigerim Ibrayeva, \\ Olga Avdienko
}

Karaganda Medical University, Karaganda, Kazakhstan

\section{Introduction}

At present, World Health Organization (WHO) demonstrates the increase worldwide morbidity and mortality from cancer in 1.5-2 times [1]. A similar trend of cancer growth is also inherent for the Republic of Kazakhstan [2].

More than 1 million people with colorectal cancer (CRC) and about 500 thousand deaths from this disease are registered in the world annually. There is an expressed geographical and ethnic variability in the spread of CRC in different countries of the world [3]. The incidence rates of CRC in Kazakhstan remain one of the highest among the CIS countries [4]. At the same time, the Karaganda region is one of the regions with a high level of cancer incidence in comparison with other regions, including CRC [2].

Gastroenterology published a study of Crockett et al., where the authors correlated the guidelines of the WHO with risk factors of potentially dangerous inflammatory colon diseases demonstrating increased risk of CRC [5]. According to Vij et al., elderly Asian patients with inflammatory bowel disease had an increased risk of colorectal cancer [6].

Despite the fact that in recent years there has been significant progress in the treatment of CRC, this disease remained one of the leading causes of death from cancer [7].

Currently, there is an evidence that inflammation is an important component of the cancer pathophysiology [8]. In response to tissue damage, the organism launches a chemical signaling cascade that can work both against abnormal cells and be used by tumor cells for their own purposes [9]. Therapeutic strategies aimed at the inflammatory process are already being considered as part of anticancer therapy, and the study of inflammatory markers at different stages are still relevant. There is a cross-link between local and systemic inflammatory processes [9], which makes it possible to use blood as the least invasive way to explore the features of the inflammatory response. Due to these reasons, there is considerable potential in the subject of studying the role of inflammatory factors, their dependence on the stage, type or extent of tumor progression.

The aim of this paper was to study the level of inflammatory cytokines in patients with CRC at different stages of the disease.

\section{Material and methods}

All groups included persons of Kazakh nationality. The permission of the KSMU Committee on Bioethics No. 305 dated May 19, 2017 was obtained to conduct research. All patients gave informed consent to participate in the study.

Group 1 - control group: 53 people aged Me [Q1; Q3] 47 [43; 57] years old, relatively healthy individuals. The surveyed people included in this group did not have relatives of the first, second and third line of relationship, suffering from malignant tumors of any location. All of these individuals underwent 
rapid fecal occult blood test and fibrocolonoduonescopy as part of the national screening.

Group 2 - comparison group: 55 persons with inflammatory bowel diseases at the age of 59.5 [53.3; 65.0] years, including Crohn's disease, intestinal polyps and polyposis of the gastrointestinal tract. During the process of forming a group, according to fibrocolonoscopy data, $62.9 \%$ of patients were diagnosed with colon polyps, and $37.1 \%$ were diagnosed with ulcerative colitis. All diagnoses were histologically confirmed.

The experimental group was represented by 309 patients aged 66 [60; 72] years, with an established diagnosis of CRC (adenocarcinoma), excluding the hereditary form (Table 1). All studied patients underwent general clinical examination at the outpatient stage. The clinical diagnosis was established according to ICD-10; the TNM classification developed by the International Union Against Cancer was used to classify the stage of cancer. Morphological verification of tumors was performed in cytological and morphological laboratories of „Regional Cancer Center” in Karaganda.

Serum biomarker blood samples were taken before the surgery. For the study the immunofluorescence method was used -panel "Human Circulation Biomarker" of the Milliplex Map series (manufactured by Millipor) and the XMap technology (Luminex), which included the simultaneous immunofluorescence determination of the following analytes on magnetic spheres: interleukin (IL)-6, IL-8, macrophage migration inhibitory factor (MIF), fibroblast growth factor (FGF-2), stem cell factor (SCF), transforming growth factor beta (TGF), tumor necrosis factor (TNF),

Table 1. Characteristics of patients in the experimental group

$\begin{array}{lcccc}\text { Patients } & \begin{array}{c}\text { Stage I } \\ (n=98)\end{array} & \begin{array}{c}\text { Stage II } \\ (n=146)\end{array} & \begin{array}{c}\text { Stage III } \\ (n=40)\end{array} & \begin{array}{c}\text { Stage IV } \\ (n=25)\end{array} \\ \text { Male } & 55(56) & 73(50) & 22(55) & 17(68) \\ \text { Female } & 43(44) & 73(50) & 18(45) & 8(32) \\ \text { Age } & 66(60 ; 72) & 66(60 ; 73) & 64(59 ; 71) & 62(52.5 ; 68)\end{array}$

Experimental group was divided according stage of colorectal cancer. Information on gender and age distribution presented in number (\%) and median (quartiles) respectively

Table 2. Serum markers in the study groups ( $\mathrm{pg} / \mathrm{ml})$

\begin{tabular}{|c|c|c|c|c|c|c|c|c|c|}
\hline Group & $n$ & IL-6 & IL-8 & MIF & FGF-2 & SCF & TGF & TNF & TRAIL \\
\hline Control & 55 & $\begin{array}{c}1.68 \\
{[0.0 ; 5.0]}\end{array}$ & $\begin{array}{c}14.24 \\
{[9.28 ; 46.56]}\end{array}$ & $\begin{array}{c}340.01 \\
{[185 ; 623.0]}\end{array}$ & $\begin{array}{c}89.54 \\
{[65.23 ; 133.0]}\end{array}$ & $\begin{array}{c}53.48 \\
{[31.52 ; 66.64]}\end{array}$ & $\begin{array}{c}9.23 \\
{[5.67 ; 12.66]}\end{array}$ & $\begin{array}{c}6.22 \\
{[4.33 ; 9.36]}\end{array}$ & $\begin{array}{c}109.53 \\
{[76.62 ; 157.1]}\end{array}$ \\
\hline Comparison & 53 & $\begin{array}{c}2.75 \\
{[0.0 ; 6.5]}\end{array}$ & $\begin{array}{c}23.10 \\
{[7.53 ; 53.97]}\end{array}$ & $\begin{array}{c}304.69 \\
{[184.9 ; 456.6]}\end{array}$ & $\begin{array}{c}93.97 \\
{[61.01 ; 140]}\end{array}$ & $\begin{array}{c}51.25 \\
{[39.6 ; 58.71]}\end{array}$ & $\begin{array}{c}9.86 \\
{[5.76 ; 17.0]}\end{array}$ & $\begin{array}{c}6.95 \\
{[4.95 ; 10.07]}\end{array}$ & $\begin{array}{c}113.17 \\
{[91.08 ; 153.26]}\end{array}$ \\
\hline I stage & 98 & $\begin{array}{c}8.1 \\
{[4.8 ; 13.8]}\end{array}$ & $\begin{array}{c}16.07 \\
{[8.99 ; 35.6]}\end{array}$ & $\begin{array}{c}188.7 \\
{[114.5 ; 361.2]}\end{array}$ & $\begin{array}{c}160.5 \\
{[93.9 ; 813.7]}\end{array}$ & $\begin{array}{c}56.92 \\
{[49.2 ; 67.3]}\end{array}$ & $\begin{array}{c}8.61 \\
{[3.92 ; 13.58]}\end{array}$ & $\begin{array}{c}7.79 \\
{[5.66 ; 10.45]}\end{array}$ & $\begin{array}{c}110.39 \\
{[67.42 ; 140.63]}\end{array}$ \\
\hline II stage & 146 & $\begin{array}{c}8.17 \\
{[2.1 ; 12.2]}\end{array}$ & $\begin{array}{c}20.11 \\
{[8.61 ; 42.89]}\end{array}$ & $\begin{array}{c}205.08 \\
{[130.2 ; 413.4]}\end{array}$ & $\begin{array}{c}134.0 \\
{[75.2 ; 220.0]}\end{array}$ & $\begin{array}{c}49.8 \\
{[33.0 ; 62.75]}\end{array}$ & $\begin{array}{c}9.53 \\
{[4.28 ; 15.80]}\end{array}$ & $\begin{array}{c}8.80 \\
{[6.11 ; 13.25]}\end{array}$ & $\begin{array}{c}94.627 \\
{[59.67 ; 129.8]}\end{array}$ \\
\hline III stage & 40 & $\begin{array}{c}7.44 \\
{[0.67 ; 15.16]}\end{array}$ & $\begin{array}{c}18.84 \\
{[10.64 ; 39.2]}\end{array}$ & $\begin{array}{c}226.6 \\
{[154.4 ; 441.4]}\end{array}$ & $\begin{array}{c}114.2 \\
{[62.15 ; 213.25]}\end{array}$ & $\begin{array}{c}51.12 \\
{[36.7 ; 59.1}\end{array}$ & $\begin{array}{c}9.70 \\
{[6.24 ; 13.2]}\end{array}$ & $\begin{array}{c}8.18 \\
{[5.63 ; 12.7]}\end{array}$ & $\begin{array}{c}101.81 \\
{[63.14 ; 135.0]}\end{array}$ \\
\hline IV stage & 25 & $\begin{array}{c}11.25 \\
{[3.67 ; 16.4]}\end{array}$ & $\begin{array}{c}50.43^{*} \\
{[21.9 ; 62.3]}\end{array}$ & $\begin{array}{c}216.2 \\
{[86.5 ; 366.3]}\end{array}$ & $\begin{array}{c}175.0 \\
{[69.3 ; 1236.9]}\end{array}$ & $\begin{array}{c}60.4 \\
{[27.9 ; 70.3]}\end{array}$ & $\begin{array}{c}10.3 \\
{[4.36 ; 19.75]}\end{array}$ & $\begin{array}{c}8.3 \\
{[5.344 ; 13.26]}\end{array}$ & $\begin{array}{c}95.8 \\
{[63.94 ; 122.26]}\end{array}$ \\
\hline $\mathrm{KW}$ & - & 0.00 & 0.05 & 0.002 & 0.0018 & 0.58 & 0.59 & 0.01 & 0.09 \\
\hline
\end{tabular}

Table 2 demonstrates concentrations of biomarkers in the following groups: control, comparison groups according stage of colorectal cancer. Concentration of biomarkers was measured in $\mathrm{pg} / \mathrm{ml}$. p-value for Kruskal-Wallis test presented for group-wide comparison. Comparison with control group with $\mathrm{p}$-value under 0.05 presented in bold. Values marked with asterisk $\left(^{*}\right)$ have $p$-value under 0.05 between stage I and stage IV according to U Mann-Whitney test tumor necrosis factor ligand superfamily member 10 (TRAIL) analytes.

Statistical analysis was performed using R statistics (Compare Groups R packages) and SPSS (IBM) programs. The nonparametric Kruskal-Wallis test was used. Mann-Whitney criteria with Holmy's correction in intergroup comparisons were applied.

\section{Results}

All presented cytokines did not have the expected significant differences between the control group and the comparison group.

The level of IL- 6 in the experimental groups of stages I-III was 4.4-4.8 times, and in 4 stages it was 6.6 times higher than in the control $(p<0.005)$ and $2.7-2.9$ times (respectively, in stage IV, 4.1 times) higher than in the comparison group (in all cases $p \leq 0.05$ ). There were not found any significant progressive deviations associated with increasing of the stage (I vs. IV, $p=0.48$ ).

The IL-8 level showed a different type of reaction. In the comparison group, a noticeable in absolute numbers, but statistically insignificant increase in the level was found. At the same time, in patients of stages I-III, the level did not differ either from the comparison group, or from the control group; a sharp increase was found in patients at IV stage ( $p=0.019$ with the control group and $p=0.022$ with the comparison group).

TNF showed moderate differences between groups, only stage 2 reached $p=0.02$ with controls and $p=0.01$ with comparison group values, but the range of change is negligible for any diagnostic decision. However, TRAIL did not demonstrate any significant differences between the groups.

An interesting point is that, the MIF level decreases in patients with stage I and II CRC, respectively, $p=0.047$ and $p=0.02$, and in stages III and IV does not differ from the values of the control group.

FGF-2 similarly significantly increases in patients with I $(p=0.002)$, II stage $(p=0.008)$ with CRC, but not in III and IV stages $(p=0.17$ and $p=0.053)$. 
Growth factors such as TGF and SCF did not differ in the presented groups. Table 2 demonstrates the values of the median and quartiles Me [Q1; Q3] for the studied markers.

\section{Discussion}

Cytokines are an extremely important part of the pathophysiological processes to which therapy can be applied. Diagnostic tools can also be developed as biomarkers differ in relative availability and contribute in diagnostics, prognosis and therapeutic monitoring [10]. In this regard, our study can serve as an introduction to further projects.

Over-expression of IL-6 is well studied in many types of cancer, and in case of CRC it is assigned an important role in the progression of the disease. IL- 6 regulates multiple signaling pathways including apoptosis, survival, proliferation, angiogenesis, invasion, and metastasis [11, 12]. In a number of studies, IL- 6 acts as a protector of cancer cells against therapy-induced DNA damage, oxidative stress and apoptosis, promoting repair [12]. In our study, IL-6 showed the substantial increase, although there was no dependence on the stage. This fact could be studied in the future, with the emphasis on the application in the diagnostic process.

IL-8, similarly to IL-6, is involved in the progression of tumor growth, promotes angiogenesis, proliferation and migration of cancer cells. The tumor can act as a source of this cytokine by itself. Its serum concentration has been shown to correlate with tumor size [13]. Serum IL-8 concentrations have been shown to be useful as a pharmacodynamic biomarker for early detection of response to immunotherapy [14]. Finally, because of the roles that IL-8 plays in tumor development, several therapeutic strategies are being developed to interfere with its function [13-15]. In our study, the data demonstrated a significant change of its level only in IV stage.

Tumor necrosis factor (TNF) is a major inflammatory cytokine characterized by its ability to induce apoptosis in tumor cells $[16,17]$. Currently, TNF being considered as a central mediator of a wide variety of biological functions, but like the rest of proinflammatory cytokines the dual impact was installed for it. In addition to apoptosis of tumor cells, which it must induce, tumor cells themselves secrete it, and then it participates in metastasis and the development of drug resistance [16-18]. However, our own data indicate that serum levels are slightly increased in all stages of CRC.

Immune cytokine tumor necrosis factor associated with apoptosis-inducing ligand (TRAIL) has received significant attention as a therapeutic agent for cancer due to its ability to selectively induce apoptosis of cancer cells without causing toxicity in vivo [19-21]. TRAIL's ability to induce cancer cell death without cellular toxicity makes it a promising therapeutic agent for a wide range of cancers [19]. Recent studies by Wu et al. have demonstrated that TRAIL can inhibit metastasis and invasion of colon cancer cells, promoting platelet apoptosis and decreasing TGF- $\beta 1$ release [21]. In our studies, we did not find a significant change in serum levels at any stages.

Macrophage migration inhibitor (MIF) is a glycoprotein that has shown itself in recent years to be an important mediator of inflammation, playing a prominent role in the pathogenesis of various types of malignant neoplasms. This marker is the initiator of the synthesis of other pro-inflammatory cytokines (IL-6, IL-8, TNF) [22-24]. In addition, MIF can promote genomic instability in tumors, as it inhibits p53 function, potentially leading to the triggering of uncontrolled proliferation. Some authors found elevated levels of MIF at CRC [23]. However, our data do not support such conclusion.

Fibroblast growth factor (FGF) is poorly understood in CRC, only recent publications dedicated to the expression of FGF on adenocarcinomas correlated with invasion in the lymph nodes, have begun to appear [25]. In our study, FGF showed noticeable differences in all groups of patients with colorectal cancer, but there was no association with stages.

Stem cell factor (SCF) is an important growth factor for hematopoietic progenitor cells with proliferative and anti-apoptotic functions [26]. Growth factors, including SCF, regulate the migration of tumor cells in almost all types of cancer [27]. Meanwhile, a number of studies suggest that significantly elevated blood SCF levels may help protect chemotherapy patients from decreased hemoglobin levels and alleviate chemotherapy-induced myelosuppression in patients with gynecologic cancer [15]. In our study, the SCF did not show significant changes comparing CRC and the control groups.

\section{Conclusions}

A better understanding of inflammation processes and the involvement of cytokines in it helps to reveal uncertain pathophysiological and clinical aspects of CRC. The main problem of measuring systemic biomarkers in an oncological process is in difference between local and serum levels of cytokines. Not every cytokine or growth factor secreted in cancer cells and exerting their action in tumor tissues manifests in the same way in serum.

Determination of the concentration of pro-inflammatory biomarkers at different stages of CRC makes it possible to reveal the dynamics of the body's inflammatory response to the tumor. As a result of our study, the most expressed reaction was shown by IL-6 starting from stage I. We can detect changes in serum levels of FGF2 and MIF only in the early stages. Inflammatory markers can hardly be initially considered as specific markers for oncopathology; however, they can be considered as part of a combined panel, in case of further successful studies. At the same time, the prospects for therapeutic action with an emphasis on growth factors and inflammatory markers are actively developing [28, 29].

We believe that further study of inflammatory cytokines is necessary to optimize the diagnosis and prognosis, in particular of colorectal cancer.

\section{Acknowledgements}

The work was done by financing the program 0.0821 (BR05236771) "Personalized approach in the management of a number of significant diseases" of The Ministry of Education and Science of the Republic of Kazakhstan.

The authors declare no conflict of interest. 


\section{References}

1. Ferlay J, Soerjomataram I, Dikshit R, et al. Cancer incidence and mortality worldwide: sources, methods and major patterns in GLOBOCAN 2012. Int J Cancer 2015; 36: 359-386.

2. Nurgaziev KS, Seitkazin GD. Indicators of the oncological service of the Republic of Kazakhstan for 2012 (statistical materials). Kazakh Scientific Research Institute of Oncology and Radiology, Almaty 2013.

3. Center MM, Jemal A, Smith RA, Ward E. Worldwide variations in colorectal cancer. CA Cancer J Clin 2009; 59: 366-378.

4. Davydov MI. Statistics of malignant neoplasms in Russia and the CIS countries in 2012-2014. M.: Publishing Group of the Russian Oncology Center, 2014.

5. Crockett S, Nagtegaal I. Terminology, Molecular Features, Epidemiology, and Management of Serrated Colorectal Neoplasia. Gastroenterology 2019; 157: 949-966.

6. Vij P, Chen D, Hsu C, et al. Racial disparities in the incidence of colorectal cancer in patients with inflammatory bowel disease. J Gastrointestinal Oncol 2019; 10: 254-258

7. Kaidarova D, Smagulova K, Yesentaeva S, Ishkinin Y, Ukolova Y, Kurmankulova A. Advanced colorectal cancer and risk factors for survival. Ann Oncol 2018; 29: mdy151.267.

8. Chen L, Deng H, Cui H, et al. Inflammatory responses and inflammation-associated diseases in organs. Oncotarget 2018; 9: 72047218.

9. Murata M. Inflammation and cancer. Environ Health Prev Med 2018; 23: 50.

10. Diakos Cl, Charles KA, McMillan DC, Clarke SJ. Cancer-related inflammation and treatment effectiveness. Lancet Oncol 2014; 15: e493-503.

11. Kumari N, Dwarakanath BS, Das A, Bhatt AN. Role of interleukin- 6 in cancer progression and therapeutic resistance. Tumour Biol 2016; 37: 11553-11572.

12. Kampan NC, Xiang SD, McNally OM, Stephens AN, Quinn MA, Plebanski M. Immunotherapeutic interleukin-6 or interleukin-6 receptor blockade in cancer: challenges and opportunities. Curr Med Chem 2018; 25: 4785-4806.

13. Alfaro C, Sanmamed MF, Rodríguez-Ruiz ME, Schalper KA, Pérez-Gracia JL, Melero I. Interleukin-8 in cancer pathogenesis, treatment and follow-up. Cancer Treat Rev 2017; 60: 24-31.

14. Waugh DJ, Wilson C. The interleukin-8 pathway in cancer. Clin Cancer Res 2008; 14: 6735-6741.

15. Nikolaou S, Qiu S, Fiorentino F, Rasheed S, Tekkis P, Kontovounisios C. Systematic review of blood diagnostic markers in colorectal cancer. Tech Coloproctol 2018; 22: 481-498.

16. Bertazza L, Mocellin S. The dual role of tumor necrosis factor (TNF) in cancer biology. Curr Med Chem 2010; 17: 3337-3352.

17. Balkwill F. Tumour necrosis factor and cancer. Nat Rev Cancer 2009; 9: 361-371.

18. Pfeffer K. Biological functions of tumor necrosis factor cytokines and their receptors. Cytokine Growth Factor Rev 2003; 14: 185-191.

19. von Karstedt S, Montinaro A, Walczak H. Exploring the TRAILs less travelled: TRAIL in cancer biology and therapy. Nat Rev Cancer 2017; 17: 352-366.

20. Guimarães PPG, Gaglione S, Sewastianik T, Carrasco RD, Langer R, Mitchell MJ. Nanoparticles for Immune Cytokine TRAIL-Based Cancer Therapy. ACS Nano 2018; 12: 912-931.

21. Wu LS, Wang XW, He W, et al. TRAIL Inhibits Platelet-Induced Colorectal Cancer Cell Invasion. J Int Med Res 2019; 47: 962-972.

22. Hu CT, Guo LL, Feng N, et al. MIF, secreted by human hepatic sinusoidal endothelial cells, promotes chemotaxis and outgrowth of colorectal cancer in liver prometastasis. Oncotarget 2015; 6: 22410-22423.

23. Guimarães Nobre CC, Galvão de Araújo JM, Araújo de Medeiros Fernandes TA, et al. Macrophage migration inhibitory factor (MIF): biological activities and relation with cancer. Pathol Oncol Res 2017; 23: 235-244.

24. Babu SN, Chetal G, Kumar S. Macrophage migration inhibitory factor: a potential marker for cancer diagnosis and therapy. Asian Pac J Cancer Prev 2012; 13: 1737-1744.
25. Jibiki N, Saito N, Kameoka S, Kobayashi M. Clinical Significance of Fibroblast Growth Factor (FGF) Expression in Colorectal Cancer Int Surg 2014; 99: 493-499.

26. Smith MA, Court EL, Smith JG. Stem Cell Factor: laboratory and clinical aspects. Blood Rev 2001; 15: 191-197.

27. Vazquez-Mellado MJ, Monjaras-Embriz V, Rocha-Zavaleta L. Erythropoietin, stem cell factor, and cancer cell migration. Vitam Horm 2017; 105: 273-296.

28. Yotsukura S, Mamitsuka H. Evaluation of serum-based cancer biomarkers: A brief review from a clinical and computational viewpoint. Crit Rev Oncol Hematol 2015; 93: 103-115.

29. Kadyrova I, Kadyrov A. Alterations of serum neurospecific proteins concentrations in patients with metabolic syndrome. Zh Nevrol Psikhiatr Im S S Korsakova 2016; 116: 92-97.

\section{Address for correspondence}

\section{Lyudmila Akhmaltdinova}

Karaganda Medical University

100017 Karaganda, Kazakhstan

e-mail: immunol.lab@gmail.com

Submitted: 24.07 .2020

Accepted: 2.11 .2020 